\title{
Quantification of Posture Induced Changes in Wearable Seismocardiogram Signals for Heart Failure Patients
}

\author{
Abdul Q. Javaid ${ }^{1}$, Sean Dowling ${ }^{2}$, Mozziyar Etemadi ${ }^{3}$, J Alex. Heller ${ }^{3}$, Shuvo Roy ${ }^{2}$, Liviu Klein ${ }^{2}$, \\ and Omer T. Inan ${ }^{1}$ \\ ${ }^{1}$ Georgia Institute of Technology, Atlanta, USA \\ ${ }^{2}$ University of California, San Francisco, USA \\ ${ }^{3}$ Northwestern University, Chicago, USA
}

\begin{abstract}
Our goal is to characterize the effects of posture-supine, seated, and standing - on the seismocardiogram (SCG) signal for patients with heart failure $(H F)$. Posture can (1) distort the SCG signal, for example due to altering the body's mechanical vibration response, and (2) affect a person's cardiovascular physiology, for example due to changes in venous return. This work focuses on characterizing the former, such that in future studies we can use the SCG to assess physiological changes in patients with HF at home. Our team has developed a circular patch ( $7 \mathrm{~cm}$ in diameter) which, when placed on the sternum, simultaneously measures the electrocardiogram (ECG) along with SCG signals in the dorso-ventral and head-to-foot directions. We recruited six HF patients thus far for this ongoing study. Each subject was asked to lie down in a supine position on a patient bed for 1 minute followed by 1 minute in each of the seated and standing postures. A novel algorithm was implemented to compare distortion in the shape of the SCG signals in the supine and seated postures as compared to the standing upright posture. The frequency domain analysis of the SCG signals revealed presence of high energy in bands greater than $8 \mathrm{~Hz}$ for supine and seated postures. Based on the findings of this paper, features can be derived to correct for posture related changes in the measured SCG signals for accurate assessment of patients with HF at home.
\end{abstract}

\section{Introduction}

Heart failure (HF) is a progressive disorder in which the heart cannot maintain an adequate supply of blood to all organs of the body. It is mainly associated with a weakened myocardium with decreased contractility. Currently, 5.7 million adults in the United States have heart failure with an associated annual health care cost of $\$ 30.7$ billion [1]. Re-admission rates to the hospital after a discharge for $\mathrm{HF}$ are $25 \%$ within the first 30 days and $45 \%$ at 6 months [2]. Continuous monitoring at home can improve the quality of life for patients with HF and can enable early detection of worsening condition. However, a thorough assessment requires information about both the electrophysiological and mechanical health of the heart. The latter cannot be provided by existing Holter-based electrocardiogram (ECG) measurement devices [3].

Ballistocardiography (BCG), a measure of the reactionary forces of the body in response to aortic ejection of blood, has re-emerged as a viable methodology for monitoring the mechanical aspects of cardiovascular diseases (CVDs) in non-clinical settings [4]. Recent research has shown that the BCG signal can be used to estimate systolic time intervals (STIs) - pre-ejection period (PEP) and leftventricular ejection time (LVET)—leading to in-depth assessment of left ventricular health [5]. A number of unobtrusive devices, which include modified bathroom-scales, beds, chairs, accelerometer based straps or patches, have been designed for measuring BCG signals from different locations on the body [4]. The nature of the body vibrations measured depend on the type and position of the sensor. Specifically, a wearable tri-axial accelerometer placed on the sternum can measure chest vibrations, called seismocardiogram (SCG), caused by both the flow of the blood in the vasculature and the closure of heart halves [6].

A major limitation in the use of BCG / SCG based sensors for home monitoring of CVDs is the fact that these cardiogenic vibrations are very sensitive to motion and postural artifacts [7]. Most of the studies with wearable SCG sensors focus on upright standing postures. However, some subjects cannot stand in an upright posture and might require measurements to be taken in supine or seated postures. These postures may not only affect the cardiovascular physiology but also add considerable artifacts to the SCG signal. Hence, it is important to analyze the effect of posture on the measured signals and identify features that can be used to differentiate posture related changes in the 

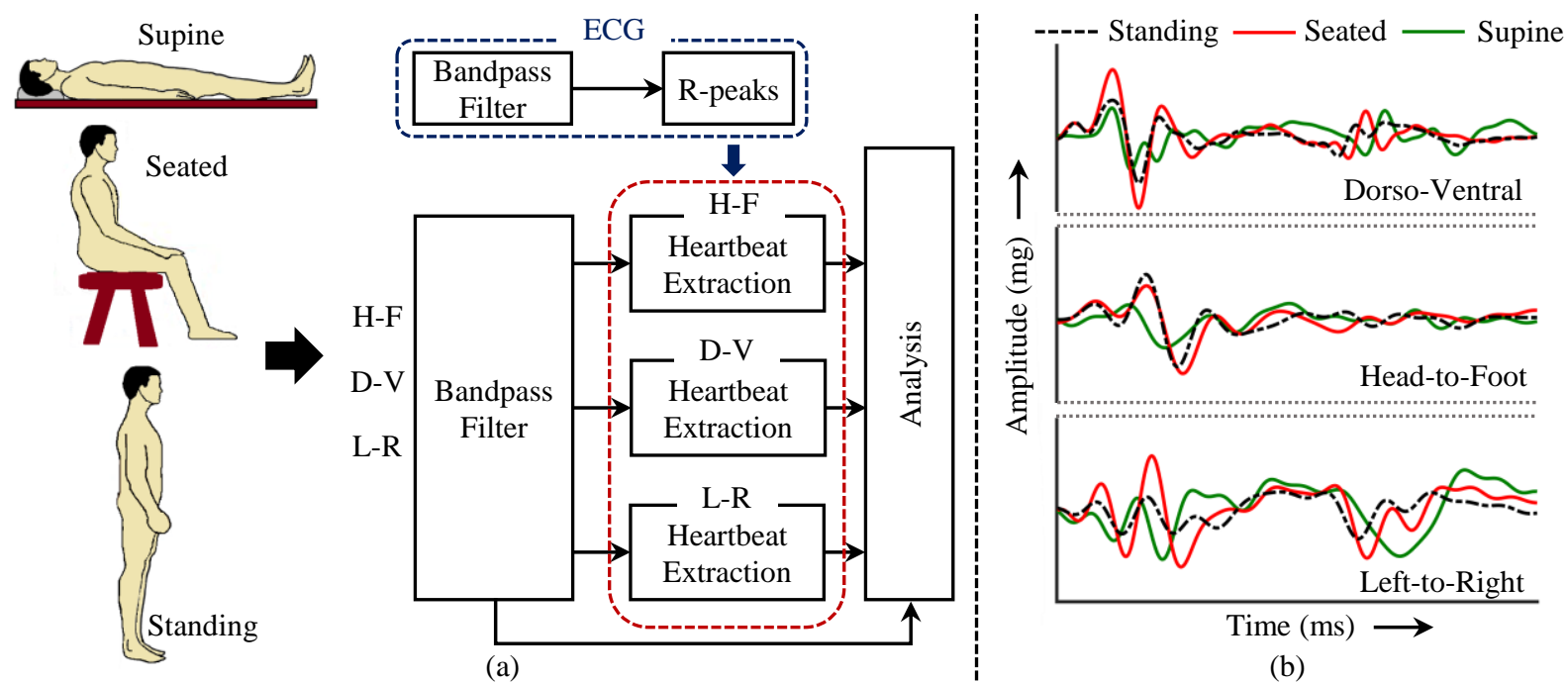

Figure 1. (a) Block diagram for the setup. The data was collected from subjects with HF in supine, seated and standing postures. (b) The acceleration signals across the 3 axes for a subject in all the three postures.

SCG signal from those caused by changes in physiology.

In this paper, we process and analyze sternal acceleration signals from a wearable patch in three different postures from patients with HF. Specifically, we analyze the power spectral density (PSD) estimates of the SCG signals along all three axes to assess frequency dependent changes in the signals in different postures. We also implement a novel algorithm to estimate the change in shape of the acceleration signals in the supine and seated postures as compared to the upright standing posture.

\section{Methods}

\subsection{Data \& Protocol}

The data for the experiment was collected from six patients with HF at the University of California, San Francisco, under an Institutional Review Board (IRB) approved protocol. Each subject was asked to wear a circular patch for SCG and ECG measurements and lay down on a patient bed in a supine position for 1-minute. This was followed by 1-minute each in an upright seated and standing posture as shown in Fig. 1 (a).

\subsection{Hardware \& Data Pre-processing}

The ECG and sternal acceleration signals (3-axis, as shown in Fig. 1 (b)) in all three postures from each subject were collected with a novel wearable patch [8] as shown in Fig. 2 (a). The wearable patch housed an ATMEGA1284P microcontroller (Atmel Corporation, San Jose, CA) and recorded data onto a micro Secure Digital (micro-SD) card (shown in Fig. 2 (b)). The ECG sensor in the patch used an analog-front-end integrated circuit

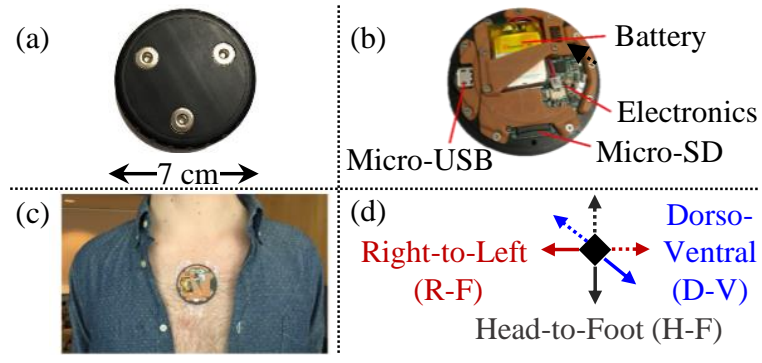

Figure 2. (a) Wearable patch $(7 \mathrm{~cm}$ in diameter) for measuring ECG and SCG signals. (b) Internal electronics in the patch. (c) The patch is adhered to the sternum with 3 pre-gelled electrodes. (d) Schematic of the tri-axial accelerometer showing different axes.

with an on-board analog-to-digital converter (ADS1291, Texas Instruments, Dallas, TX) while BMA280 (Bosch Sensortec GmbH, Reutlingen, Germany) was selected for the accelerometer. The patch was placed on the sternum of each subject using three electrodes as shown in Fig. 2 (c).

The ECG and accelerometer signals were band-pass filtered using finite impulse response (FIR) filters (Kaiser window, cut-off frequencies: $0.8-40 \mathrm{~Hz}$ for the ECG, and, $0.8-35 \mathrm{~Hz}$ for the dorso-ventral (D-V) and $0.8-20 \mathrm{~Hz}$ for the head-to-foot (H-F) and left-to-right (L-R) components of acceleration signals) as shown in Fig. 1 (a). The three axes of the accelerometer are shown in Fig. 2 (d).

\subsection{Quantification of Shape Distortion}

A novel algorithm was implemented to analyze changes in the shape of accelerations signals during the supine and seated postures as compared to the upright standing pos- 


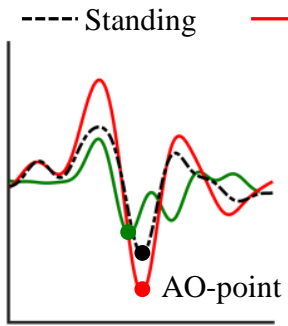

(a)

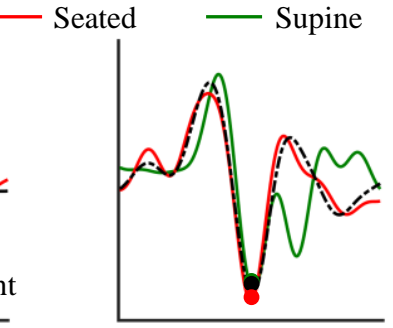

(b)
Figure 3. Analyzing the change in shape for the $\mathrm{D}-\mathrm{V}$ heartbeats in the 3 postures. (a) The AO-point was detected in the $\mathrm{D}-\mathrm{V}$ heartbeats as the global maximum or minimum with highest absolute amplitude. (b) The heartbeats are aligned using the location of the AO-point and re-sampling with cubic interpolation.

ture. Once all the signals were filtered, the R-peaks $R_{i}(i$ is the peak index) in the ECG signal were detected with an automated algorithm. With $R_{i}$ as reference and $l$ as the frame size, $R_{i}+l$ frames, called 'heartbeats', were extracted from the D-V, H-F and L-R signals from each subject. The frame size $l$ was estimated as the minimum R-R interval in the ECG for the corresponding posture. The heartbeats extracted in each posture were averaged to obtain one time-averaged trace for each posture.

The feature corresponding to the opening of the aortic valve (AO-point) in the $\mathrm{D}-\mathrm{V}$ heartbeat from the standing upright posture of a subject was detected as the maximum or minimum with the highest absolute amplitude value in the first $200 \mathrm{~ms}$ of the heartbeat and its position was denoted by $f_{o}$. If the AO-point was detected as the global minimum in the $\mathrm{D}-\mathrm{V}$ trace of the standing posture for a subject, then the AO-point in the supine or seated postures was also chosen as the global minimum for that subject. A similar approach was used if the maximum was selected. After the correct detection of the AO-point in each posture, only the first $f_{o}+\alpha \mathrm{ms}$ frame was extracted from the heartbeat in each posture $(\alpha=100 \mathrm{~ms})$ as the first half of the acceleration heartbeat along any axis has the highest signal-to-noise ratio. Let this extracted portion be denoted by $d_{k}$, where $k$ represents posture ( $k \in[$ stand, seated, supine]). In order to account for the change in ECG R-peak-to- $f_{o}$ interval (PEP) in different postures, the $d_{k}$ extracted frames from the seated and supine postures were aligned with the standing posture frame using the location of the AO-points as shown in Fig. 3 (a). Specifically, the portions of the supine or seated frame before and after the AO-point were re-sampled using cubic interpolation so the total length of the frame was equal to that of the corresponding frame in the standing posture. Once the frames from the supine and seated postures were aligned with the standing posture signal (as shown in Fig. 3 (b)), all the signals were standardized by subtracting the mean and dividing by the standard deviation. The root mean square error (RMSE), was calculated between the supine and standing posture and also between seated and standing posture for each subject.

A similar approach was used for the H-F heartbeats and the L-R heartbeats. However, rather than detecting the AOpoint, the J-peak in the H-F and L-R heartbeats was detected as the highest peak in the first $300 \mathrm{~ms}$ portion of the signal. For the supine and seated postures, the J-peak was selected as the highest peak $100 \mathrm{~ms}$ around the position of the J-peak in the standing posture for that subject. The RMSE was calculated for the supine and seated postures compared to the standing posture for both H-F and L-R heartbeats for all subjects.

\subsection{Frequency Domain Analysis}

To analyze the frequency domain difference in the measured acceleration signals, the Welch's periodogram method was used to obtain PSD estimates for D-V, H-F and L-R signals. However, PSD values were calculated on the filtered signals and not on the extracted heartbeats. The PSD estimates were normalized by the maximum value in the $0-10 \mathrm{~Hz}$ band. The mean and standard deviation of PSD values were calculated for all subjects in the $0-20 \mathrm{~Hz}$ band.

\section{Results \& Discussion}

\subsection{Shape Analysis}

The shape error for seated and supine positions for all subjects is summarized in Fig. 4. It can be observed that the RMSE for the seated posture is smaller for all the axes of the acceleration signals. The minimum RMSE was observed for $\mathrm{D}-\mathrm{V}$ heartbeats in the seated posture. It can be concluded from the results that $\mathrm{D}-\mathrm{V}$ signals in the seated posture undergo the least shape distortion as compared

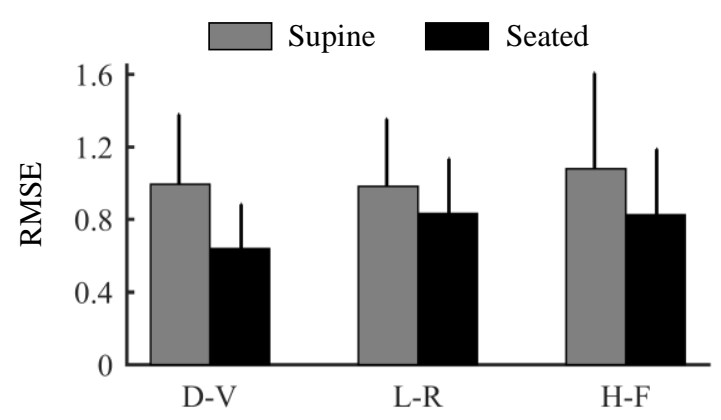

Figure 4. RMSE results for quantifying shape distortion in the heartbeats from 3 axes of the accelerometer in the supine and seated postures. 

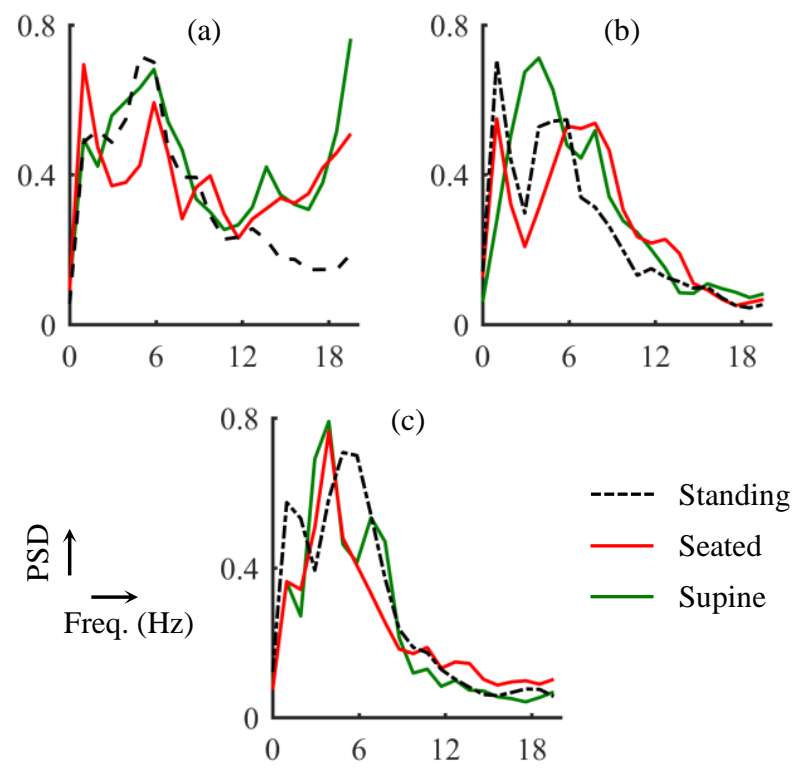

Figure 5. Frequency domain analysis. (a) Mean PSD estimates for $\mathrm{D}-\mathrm{V}$ acceleration signals in the three postures. (b) Mean PSD estimates for H-F acceleration signals in the three postures. (c) Mean PSD estimates for L-R acceleration signals in the three postures.

to corresponding signals in the upright standing posture. However, H-F and L-R signals show similar results.

\section{2. $\quad$ PSD Based Differences}

The initial PSD results indicate that the D-V vibrations in both the supine and seated positions are accompanied with an increase in energy in the high frequency band (greater than $10 \mathrm{~Hz}$ ) as shown in Fig. 5 (a). The H-F vibrations show increased energy in the $6-12 \mathrm{~Hz}$ band for the supine and seated positions. These results are consistent with our previous findings with healthy subjects that the changes in SCG morphology-specifically, increased higher frequency vibration modes in addition to the primary mode in the $1-5 \mathrm{~Hz}$ range-are more associated with body mechanics rather than physiologic changes [9]. The L-R axis signals, however, do not show increased energy in higher frequency bands.

\section{Conclusion}

In this paper, we have presented our initial findings on how different postures can affect the shape and frequency content of SCG signals of patients with HF. The results indicate that features can be extracted from the SCG signals to assess changes in posture. Our future work will focus on correcting for these changes and analyzing the postural effects on the physiology of patients with HF at home.

\section{Acknowledgements}

Research reported in this publication was supported in part by the National Institute on Aging of the National Institutes of Health (NIH) under Award Number R56AG048458 and the National Institute of Biomedical Imaging and Bioengineering of the NIH under 1U01EB018818-01. The content is solely the responsibility of the authors and does not necessarily represent the official views of the National Institutes of Health.

\section{References}

[1] Mozaffarian, Dariush, et al. "Executive Summary: Heart Disease and Stroke Statistics-2016 Update: A Report From the American Heart Association." Circulation 133.4 (2016).

[2] Gheorghiade, Mihai, et al. "Congestion in acute heart failure syndromes: an essential target of evaluation and treatment." The American journal of medicine 119.12 (2006): S3-S10.

[3] Oresko, Joseph J., et al. "A wearable smartphone-based platform for real-time cardiovascular disease detection via electrocardiogram processing." IEEE Transactions on Information Technology in Biomedicine 14.3 (2010): 734 740 .

[4] Inan, Omer T., et al. "Ballistocardiography and seismocardiography: A review of recent advances.” IEEE journal of biomedical and health informatics 19.4 (2015): 1414-1427.

[5] Boudoulas, H. "Systolic time intervals." European heart journal 11.suppl I (1990): 93-104.

[6] Tavakolian, Kouhyar, et al. "Myocardial contractility: a seismocardiography approach." 2012 Annual International Conference of the IEEE Engineering in Medicine and Biology Society. IEEE, 2012.

[7] Javaid, Abdul Qadir, et al. "Elucidating the Hemodynamic Origin of Ballistocardiographic Forces: Toward Improved Monitoring of Cardiovascular Health at Home." IEEE Journal of Translational Engineering in Health and Medicine 4 (2016): 1-8.

[8] Etemadi, Mozziyar, et al. "A Wearable Patch to Enable Long-Term Monitoring of Environmental, Activity and Hemodynamics Variables." IEEE transactions on biomedical circuits and systems 10.2 (2016): 280-288.

[9] Javaid, Abdul Qadir, et al. "Quantifying and Reducing Posture-Dependent Distortion in Ballistocardiogram Measurements." IEEE journal of biomedical and health informatics 19.5 (2015): 1549-1556.

Address for correspondence:

Omer T. Inan

Technology Square Building, 85th Fifth St NW, Suite 412

Atlanta, GA 30308, USA.

inan@gatech.edu 